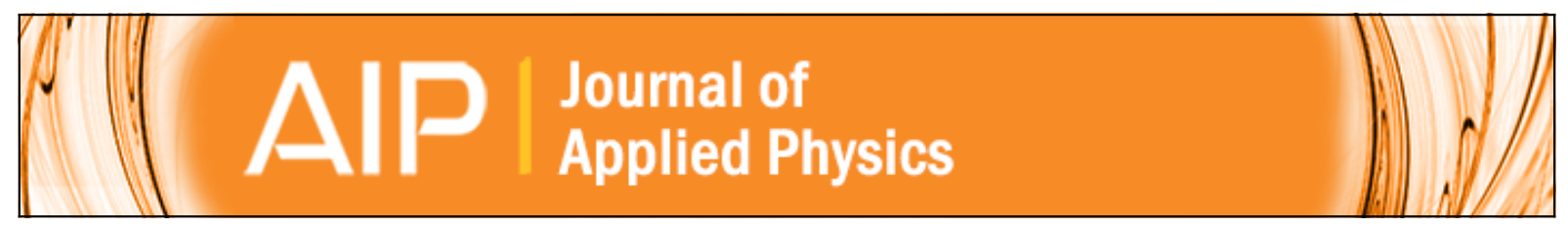

\title{
Size and dimensionality effect in single-impurity Anderson model
}

Feng Chen and Nicholas Kioussis

Citation: Journal of Applied Physics 83, 6429 (1998); doi: 10.1063/1.367667

View online: http://dx.doi.org/10.1063/1.367667

View Table of Contents: http://scitation.aip.org/content/aip/journal/jap/83/11?ver=pdfcov

Published by the AIP Publishing

\section{Articles you may be interested in}

Crystal structure effect on the ferromagnetic correlations in $\mathrm{ZnO}$ with magnetic impurities

J. Appl. Phys. 104, 103906 (2008); 10.1063/1.3028262

Non-Fermi-liquid properties of three-impurity Anderson models

AIP Conf. Proc. 918, 342 (2007); 10.1063/1.2752007

A diagrammatic solution of the single-impurity Anderson model in the Kondo regime AIP Conf. Proc. 918, 272 (2007); 10.1063/1.2751994

Effect of f-band dispersion on the magnetic properties of periodic Anderson lattice model J. Appl. Phys. 95, 7195 (2004); 10.1063/1.1676024

The numerical approach to the correlated electron problem: quantum Monte Carlo methods AIP Conf. Proc. 678, 117 (2003); 10.1063/1.1612393

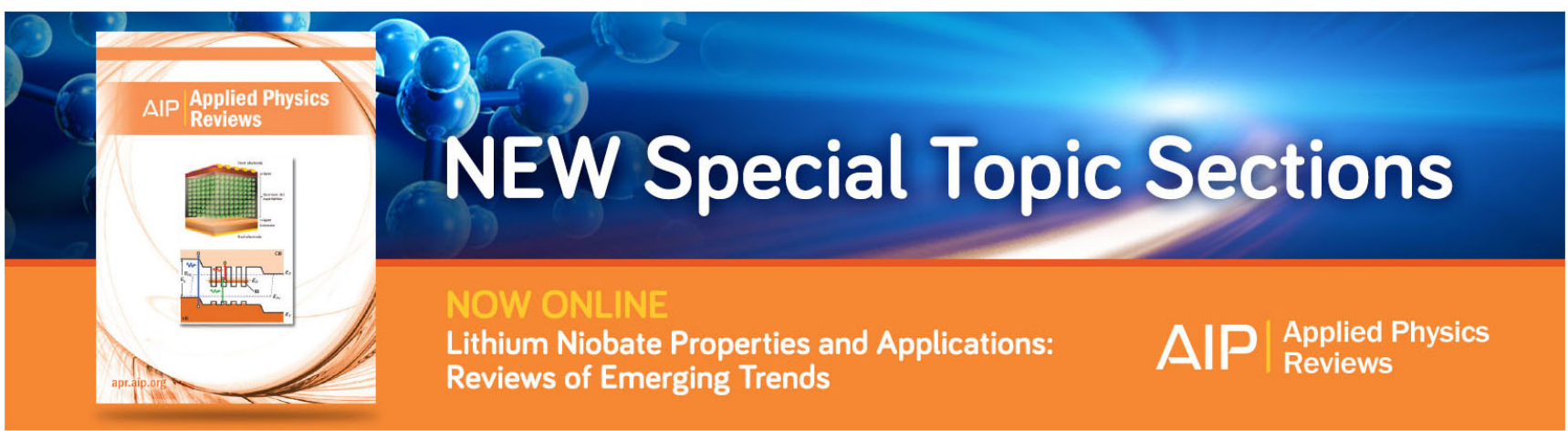




\title{
Size and dimensionality effect in single-impurity Anderson model
}

\author{
Feng Chen and Nicholas Kioussis ${ }^{\text {a) }}$ \\ Department of Physics, California State University Northridge, Northridge, California 91330-8268
}

The dimensionality and the size effect on the magnetic properties of the single-impurity symmetric Anderson model in three-, two-, and one-dimensional lattices has been studied using the quantum Monte Carlo method. We have considered clusters with odd number of sites with the impurity placed at the center of the cluster and have employed open boundary conditions. In 3D and 1D lattices, we find that at sufficiently low temperatures, the system can be in either a spin compensated or noncompensated state as the cluster size is reduced. On the other hand, in 2D the system is always in a spin-compensated state independent of the cluster size. These results imply physics beyond that contained in the simplest Kondo model and may explain the discrepancy between two recent experimental results. (C) 1998 American Institute of Physics. [S0021-8979(98)30811-7]

The Kondo effect involves the interaction of a localized moment with an electron gas and is relevant to the behavior of dilute magnetic alloys, as well as concentrated systems such as the heavy-fermion materials. The ground state of the single-impurity Kondo problem is a singlet in which the local moment is "compensated" by the conduction electrons due to the antiferromagnetic exchange interaction between the local moment and the conduction electrons. ${ }^{1}$ Several calculations predict that the length scale of the compensation cloud is of the order $R_{K} \approx h v_{F} / 2 \pi k_{B} T_{K} \cdot{ }^{2}$ On the other hand, several experiments suggest that the length scale is of an order of magnitude smaller than the theoretical value or that the polarization cloud may not even exist. ${ }^{3}$ Thus surprisingly, despite the large amount of work on the Kondo problem, it appears that the question of length scale is far from settled.

Nearly all of the work on the single-impurity Kondo problem has involved bulk, i.e., three-dimensional systems. However, in recent years the behavior and the properties of the spin-compensation cloud in lower-dimensional (quasione and quasi-two dimensional) systems have attracted much attention both experimentally ${ }^{4-8}$ and theoretically. ${ }^{9,10}$ Since a length scale $\left(R_{K}\right)$ is associated with the Kondo effect, one might expect the Kondo behavior to be different in a small system (as compared to a "bulk" sample), provided that one or two dimensions are small compared to the relevant length scale $R_{K}$. The experimental results can be summarized as follows: (i) A large size dependence was found for $\mathrm{Au}(\mathrm{Fe})$ and $\mathrm{Cu}(\mathrm{Fe})$ thin films (for film thickness less than $2000 \AA$ ), where the Kondo contribution to the resistivity is suppressed; ${ }^{4}$ (ii) no size dependence of the Kondo resistivity was found in $\mathrm{Au}(\mathrm{Fe})$ wires when the width dependence of the electron-electron interaction contribution to the resistivity was taken into account; ${ }^{7}$ and (iii) the Kondo temperature $T_{K}$ shows no size dependence. ${ }^{6}$

The purpose of this paper is to investigate the effect of: (1) the size of the system and (2) the dimensionality on the ground-state properties of the single-impurity Anderson model. Although the effect of size on the Friedel resonance

\footnotetext{
a)Electronic mail: nkioussi@newton.csun.edu
}

was studied recently by Bergmann, ${ }^{12}$ to our knowledge, the effect of dimensionality on the ground-state properties has not been studied theoretically. In reduced dimensions, systems may exhibit anomalous properties. For example, the Van Hove singularities in the density of states and the nested Fermi surface in a two-dimensional lattice or the chargedensity instability in one dimension may effect the ground state properties of the Kondo problem.

Quantum Monte Carlo methods have been used previously to study the single-impurity Anderson model and have provided great insight into the magnetic properties. ${ }^{13,14}$ However, while in all previous simulations the magnetic impurity is placed in a three-dimensional infinite sea of conduction electrons, in the present work the conduction electrons are confined on a regular lattice. In order to study the size effect, we employ open boundary conditions and place the magnetic impurity in the middle of the lattice. In open boundary conditions, conduction electrons that reach the boundary sites can hop only back to interior sites. We have changed the dimensionality and the size of the lattice and have studied the local moment and the temperature dependent impurity susceptibility. The results of the numerical simulations indicate that the properties of the ground state are quite different than those for the bulk systems depending on the dimensionality and the size of the lattice, and may explain the discrepancy between two recent experimental results. ${ }^{5-7}$

The single-impurity Anderson model is

$$
\begin{aligned}
H= & -t \sum_{\langle i, j\rangle \sigma}\left(c_{i \sigma}^{+} c_{j \sigma}+H . c .\right)+E_{d} \sum_{\sigma} d_{\sigma}^{+} d_{\sigma}+U n_{d \uparrow} n_{d \downarrow} \\
& +V\left(c_{i_{0} \sigma}^{+} d_{\sigma}+\text { h.c. }\right)
\end{aligned}
$$

where $c_{i, \sigma}^{+}$creates a conduction electron on site $i$ with spin $\sigma, d_{\sigma}^{+}$creates a localized electron on the impurity site $i_{0}$ with spin $\sigma, E_{d}$ is the energy of a singly occupied impurity level, $U$ is the Coulomb interaction between two $d$ electrons on the impurity site, and $V$ is the on-site hybridization energy between the conduction-electron orbital and the impurityelectron orbital.

We have used a finite-temperature Monte Carlo technique with an exact updating procedure. A typical Monte 
Carlo run involved 5000 sweeps through the lattice and the statistical error for the quantity measured was negligible except where shown. The finite time slice $\delta \tau$ introduces a systematic error. Simulations with $\delta \tau=0.125$ and 0.25 give results which differ only by a few percent. As a check on our Monte Carlo algorithm we have done extensive comparison with exact diagonalization results for an impurity interacting with a two-site conduction-electron lattice. We consider the symmetric case with $E_{d}=-U / 2$, which is the most favorable for the formation of an impurity magnetic moment, and the case of half-filled conduction band and one localized $d$ electron. This choice ensures particle-hole symmetry. The Monte Carlo simulations were carried out in the intermediate parameter regime, where the correlation effects are comparable in size to the hybridization interaction, i.e., $U / t=V / t=1$. In the intermediate regime Monte Carlo simulations provide essentially exact results, whereas analytic approaches are most likely to fail or be inaccurate.

In order to investigate the effect of size and dimension on the spin-compensation cloud, we have calculated the impurity local moment:

$$
\left\langle\left(\sigma_{z}^{d}\right)^{2}\right\rangle=\left\langle\left(n_{d \uparrow}-n_{d \downarrow}\right)^{2}\right\rangle .
$$

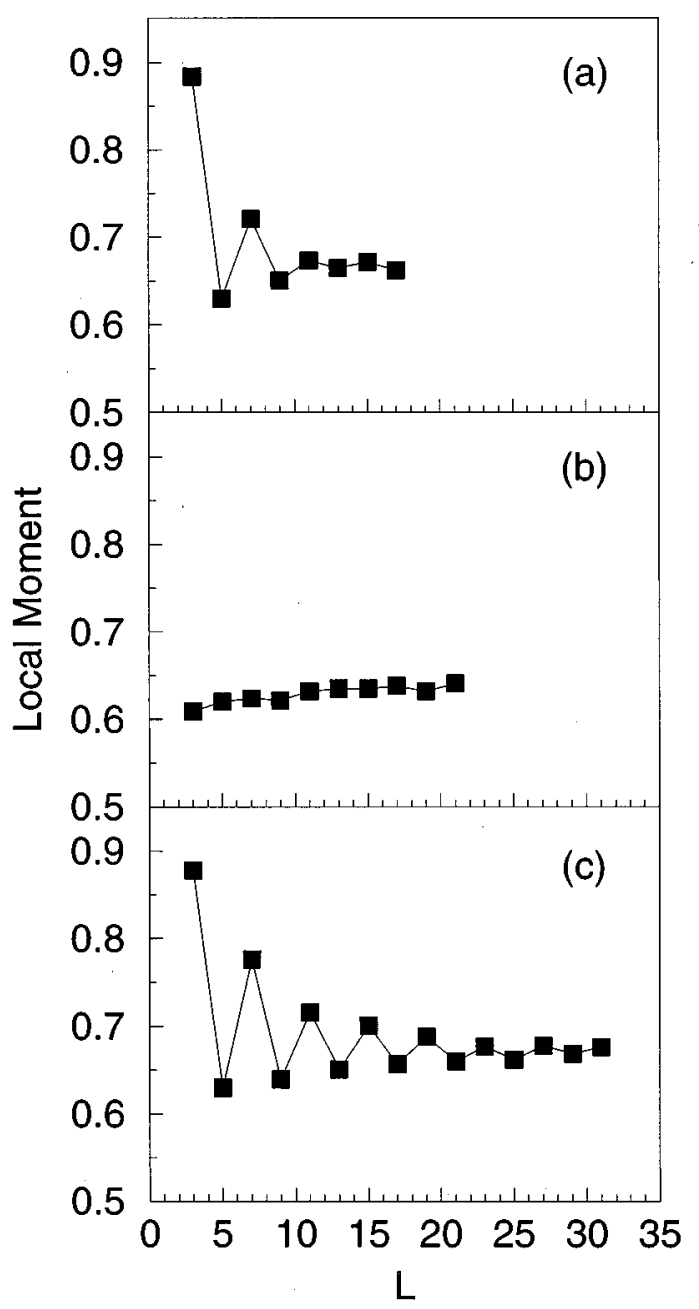

FIG. 1. Local magnetic moment, $\left\langle\left(\sigma_{z}^{d}\right)^{2}\right\rangle$, vs cluster size $L^{n}$ for $U=1, V$ $=1$, and $\beta=16$ in (a) a 3D cubic lattice, (b) a 2D square lattice, and (c) a 1D lattice.
In Fig. 1 we show the local moment, $\left\langle\left(\sigma_{z}^{d 2}\right)\right\rangle$, versus the cluster size ( $L^{n}$ for $n$ dimension) for three [Fig. 1(a)] two, [Fig. 1(b)] and one dimension [Fig. 1(c)] at low temperatures $\beta=16 / t$. One can see that the local moment in one and three dimensions oscillates as the lattice size is reduced. This result indicates that for 3D and $1 \mathrm{D}$ there are two different types of ground states, a compensated one (for $L=5$, nine sites) and an uncompensated one (for $L=3$, seven sites). The local magnetic moment associated with the uncompensated ground state is larger than that of the compensated state and and shows a larger size effect. The moment decreases exponentially with lattice size, $\mu \propto e^{-L / L_{0}}$, where $L_{0}$ is 7.6 for $3 \mathrm{D}$ and 12.8 for the 1D lattice. On the other hand, the local moment in 2D varies monotonically with lattice size, indicating the existence of one type of ground state only, the compensated one.

The size effect in $1 \mathrm{D}$ can be understood by examining the Anderson-Hamiltonian for $U=0$ which can be diagonalized exactly. The eigenfunctions in 1D can either be of odd or even parity depending on the number of sites in the system. The eigenfunctions of even (odd) parity have a large (small) spectral weight on the impurity site which gives rise

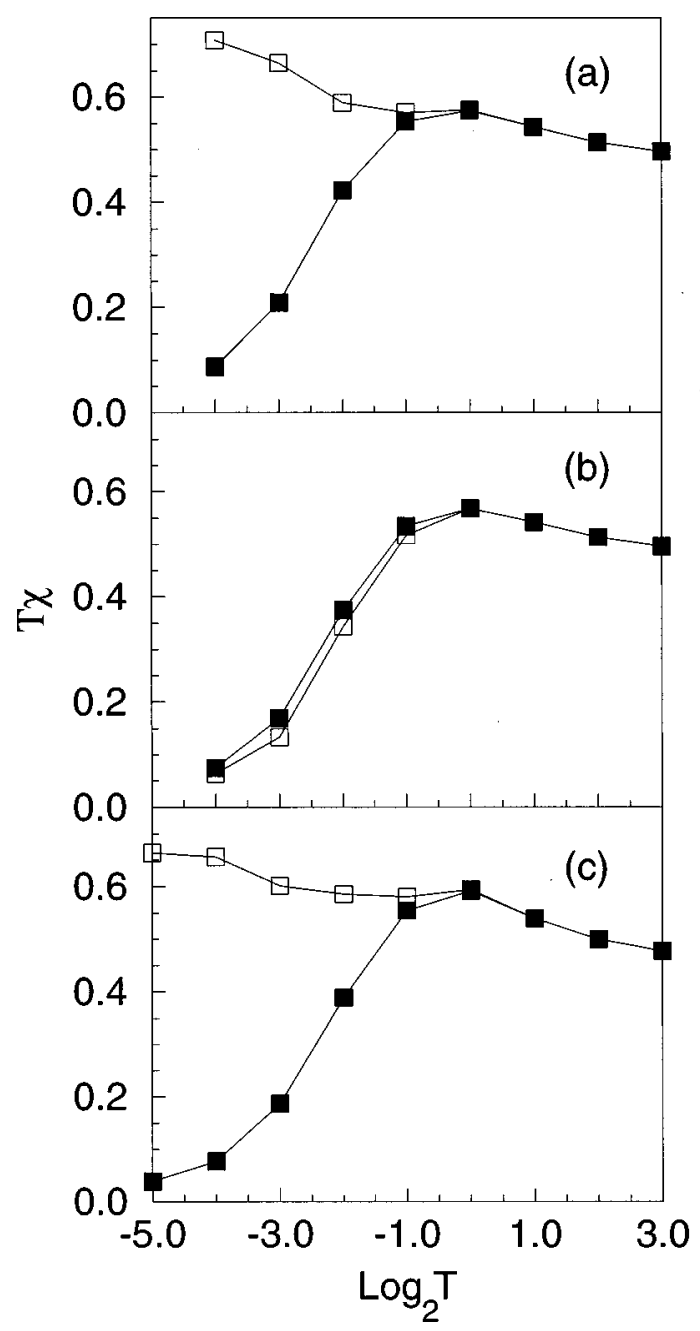

FIG. 2. Impurity magnetic susceptibility vs $\log _{2} T$ for $U=1$ and $V=1$ for (a) 3D lattice, (b) 2D lattice, and (c) 1D lattice, for the cluster size $L^{n}$ $=3^{n}$ and $L^{n}=5^{n}$, respectively. 
to a strong (weak) antiferromagnetic interaction between the local and conduction electrons. Thus, for cluster size of $L$ $=3,7, \ldots$ the states at the Fermi energy are of odd parity giving rise to an "uncompensated" state, whereas for $L$ $=5,9, \ldots$ the states at the Fermi energy are of even parity giving rise to a "compensated" ground state.

In order to gain further insight of the nature of the ground state, we have calculated the impurity $d$-spin magnetic susceptibility

$$
T_{\chi}=\int_{0}^{\beta} d \tau\left\langle\sigma_{z}^{d}(\tau) \sigma_{z}^{d}(0)\right\rangle,
$$

as a function of temperature. In Fig. 2, we plot the "effective moment,' $T_{\chi}$, vs $\log _{2} T$ for three [Fig. 2(a)], two [Fig. 2(b)], and one dimension [Fig. 2(c)] for the cluster size $L^{n}=3^{n}$ and $L^{n}=5^{n}$ ( $n$ is the dimension), respectively. If the ground state is compensated, the effective moment, $T_{\chi}$, should decrease to zero at lower temperatures, signaling the quenching of the local moment. In Fig. 2, we plot these calculations with $L$ $=3$ and 5 . It is clear that in $3 \mathrm{D}$ and $1 \mathrm{D}$, the ground state can be either a compensated $(L=5)$ state or an uncompensated ( $L=3$ ) state, while the ground state in the 2D lattice can only be a compensated state.

In order to check the effect of boundary conditions on the ground state properties, we have also carried out Monte Carlo simulations for systems with even number of sites employing periodic and antiperiodic boundary conditions. The results for the size and dimensionality effect on the ground state properties are similar to those reported in this work for the case of systems with odd number of sites with open boundary conditions.

The absence of a size effect in our calculations for the 2D lattice is consistent with the experimental results of Chandrasekhar et $a l^{7}$ On the other hand, the presence of a size effect in $3 \mathrm{D}$ is consistent with the experimental results of Giordano et al. ${ }^{5,6}$ The Kondo temperature inferred from the temperature dependence of the impurity magnetic susceptibility does not depend on the system size in 1D, 2D, and
3D lattices, which is consistent with the experimental results. $^{5-8}$ It is interesting to consider the case of a magnetic impurity doped quantum dot, although no experimental results are currently available. Our results in 1D suggest that the ground state of the quantum dot can be a compensated or an uncompensated state as the size of the wire is reduced.

In conclusion, the dimensionality of the conduction electrons affect their interaction with the impurity resulting in a compensated or an uncompensated ground state for the case of 3D and 1D depending on the lattice size, and to a compensated ground state for the case of $2 \mathrm{D}$. The dimensionality and size effect are consistent with the experimental results for the Kondo resistivity anomalies in doped films and wires. We predict that there should be a large size size effect in doped quantum dots.

The research at California State University Northridge (CSUN) was supported through the National Science Foundation under Grant No. DMR-9531005 and the Office of Research and Sponsored Projects at CSUN.

${ }^{1}$ K. Fischer, in Springer Tracts in Modern Physics, edited by G. Höhler (Springer, Berlin, 1970), Vol. 54, p. 1.

${ }^{2}$ H. Ishii, Prog. Theor. Phys. 55, 1373 (1976).

${ }^{3}$ C. P. Slichter, in Magnetism and Magnetic Materials, AIP Conf. Proc. No. 29, edited by J. J. Becker, G. H. Lander, and J. J. Rhyne (AIP, New York, 1976), p. 306.

${ }^{4}$ M. A. Blachly and N. Giordano, Phys. Rev. B 51, 12537 (1995).

${ }^{5}$ G. Chen and N. Giordano, Phys. Rev. Lett. 66, 209 (1991).

${ }^{6}$ M. A. Blachly and N. Giordano, Phys. Rev. B 49, 6788 (1994).

${ }^{7}$ V. Chandrasekhar, P. Santhanam, N. A. Penebre, R. A. Webb, H. Vloeberghs, C. Van Haesendonck, and Y. Bruynseraede, Phys. Rev. Lett. 72, 2053 (1994).

${ }^{8}$ J. F. DiTusa, K. Lin, M. Park, M. S. Isaacson, and J. M. Parpia, Phys. Rev. Lett. 58, 678 (1992).

${ }^{9}$ O. Ujsaghy, A. Zawadowski, and B. L. Gyorffy, Phys. Rev. Lett. 76, 2378 (1996).

${ }^{10}$ I. Martin, Y. Wan, and P. Philips, Phys. Rev. Lett. 78, 114 (1997).

${ }^{11}$ K. G. Wilson, Rev. Mod. Phys. 47, 773 (1975).

${ }^{12}$ G. Bergmann, Phys. Rev. Lett. 67, 2545 (1991).

${ }^{13}$ J. E. Hirsch and R. M. Fye, Phys. Rev. Lett. 56, 2521 (1986).

${ }^{14}$ J. E. Gubernatis, J. E. Hirsch, and D. J. Scalapino, Phys. Rev. B 35, 8478 (1987) 